\title{
Traumatismo orofacial em crianças: conhecimento de profissionais de creches de um município brasileiro
}

\section{Orofacial trauma in children's day care professional knowledge of a brazilian city}

\author{
Andreza Cristina de Lima Targino Massoni' \\ Izabelle Cristinne Freitas do Ó Leite Dóia" \\ ÉRIKA PORTOIII \\ Lorena Marques da Nóbrega"l \\ Sérgio d’Ávila Lins BeZerra CaValcantI"I \\ Ana Flávia GranVILle-Garcia III
}

\begin{abstract}
RESUMO
Introdução: O traumatismo orofacial é uma situação de urgência odontológica que exige do profissional atendimento rápido. Considerando que vários tipos de traumas podem ocorrer no ambiente escolar, os funcionários destas Instituições são os primeiros a terem a oportunidade de conduzir a assistência a ser prestada. Aspecto que pode influenciar diretamente no prognóstico do caso. Objetivo: Avaliar o conhecimento de profissionais de creches municipais na cidade de Patos/PB, sobre traumatismo orofacial em crianças. Materiais e Método: 121 profissionais de creches públicas responderam a um questionário. Os dados foram analisados pela estatística descritiva, através dos Testes Qui-quadrado de Pearson e Exato de Fisher, com nível de significância de $5 \%$. Resultado: A maioria dos participantes informou não ter recebido orientação sobre traumatismo orofacial $(89,3 \%)$ e não se sentir preparado para prestar socorro às crianças $(47,1 \%)$, destacando o interesse em receber informações sobre o tema $(97,5 \%)$. Estas variáveis não estiveram associadas à escolaridade dos investigados $(p>0,05)$. Estes, ainda afirmaram ter vivenciado experiências de traumatismo, estando associados principalmente às quedas $(55,1 \%)$, sendo as lacerações nos lábios os tipos mais relatados $(46,3 \%)$. Em casos de assistência à criança, o Professor foi destacado como o responsável $(59,5 \%)$. Entre os cuidados citados diante de traumatismo, os participantes relataram que é adequado limpar com gaze umedecida em soro fisiológico o local afetado, bem como aplicar gelo $(40,7 \%)$. Conclusão: Os profissionais investigados apresentaram conhecimento limitado em relação ao traumatismo orofacial em crianças com idade pré-escolar, o que desperta para a necessidade de uma abordagem educativa junto a estes profissionais sobre o tema.
\end{abstract}

Palavras-chave: Educação infantil; pré-escolar; traumatismos faciais; cavidade oral; dente decíduo.

\begin{abstract}
Introduction: The orofacial trauma is a dental urgency situation that requires the professional fast service. Whereas various types of trauma can occur at school, employees of these institutions are the first to have the opportunity to drive the assistance to be provided. Aspect that can directly influence the outcome of the case. Objective: To evaluate the knowledge of professionals from day-care centers in a city in northeastern Brazil on orofacial trauma. Method: 121 professionals from the public day-care centers responded to a questionnaire. Data were analyzed by descriptive statistic, using the Chi-square test and Fisher exact test, with significance level of $5 \%$ and the subjective. Results: Most participants reported not having received guidance on orofacial trauma $(89.3 \%)$ and did not feel prepared to provide relief to children (47.1\%), highlighting the interest in receive information about this subject (97.5\%). These variables were not associated with the school investigated $(p>0.05)$. Although claimed to have experienced traumatic experiences, associated mainly to falls $(55.1 \%)$, and lacerations to his lips the most reported types (46.3\%). In cases of trauma to child care, teacher was highlighted as responsible for providing first aid (59.5\%). Among trauma care mentioned before, participants reported that it is appropriate clean with gauze moistened with saline the affected site and apply ice (40.7\%). Conclusion: The professionals surveyed had limited knowledge in relation to orofacial trauma in children of preschool age, which arouses the need for an educational approach with these professionals on the theme.

Keywords: Early childhood education, preschool, facial trauma, oral cavity; deciduous tooth.
\end{abstract}

\footnotetext{
Universidade Estadual da Paraíba (UEPB), Campina Grande/PB - Brasil

"Faculdades Integradas de Patos (FIP), Patos/PB - Brasil

"'Universidade Estadual da Paraíba (UEPB), Campina Grande/PB - Brasil
} 


\section{INTRODUÇÃO}

O traumatismo orofacial é uma situação de urgência odontológica que exige do profissional um atendimento rápido. Este agravo acomete crianças precocemente; em geral ainda na primeira infância; o que as torna bastante susceptíveis. Entre os motivos que favorecem a exposição de crianças em idade pré-escolar ao traumatismo orofacial, citamse o crescimento e o desenvolvimento físico e psíquico, pois nessa fase elas têm inicio aos primeiros passos, bem como a coordenação motora ainda está se estabelecendo ${ }^{1}$.

Um quarto de todas as crianças que frequentam escolas e quase um terço dos adultos sofreu traumatismo dental em algum momento da vida ${ }^{2}$. No Brasil, a prevalência de traumatismos orofaciais na dentição decídua varia de 14 a $36 \%$, e poucas crianças chegam aos quatro anos de idade sem nunca terem sofrido algum tipo de trauma ${ }^{3}$.

Considerando que vários tipos de trauma podem ocorrer no ambiente escolar, durante atividades esportivas e recreativas entre crianças, que os funcionários destas Instituições são os primeiros a terem contato com a criança acidentada, que este aspecto pode influenciar no prognóstico da lesão ${ }^{4}$ e que, nem sempre esta assistência é adequada, por falta de treinamento destes profissionais ${ }^{5,6,7,8 \text {, }}$ 9 , é objetivo deste estudo avaliar o treinamento e conhecimentos sobre traumatismo orofacial, de profissionais que atuam em creches municipais da cidade de Patos/PB.

\section{Metodologia}

Tratou-se de uma pesquisa descritiva, quantitativa, do tipo transversal; cuja população compreendeu Diretores e Educadores que trabalham em Creches municipais, desempenhando a função de cuidadores de crianças em idade pré-escolar, na cidade de
Patos/PB, situada no Nordeste Brasileiro. Segundo informações da Secretaria Municipal de Educação, a cidade conta com 11 Creches municipais, as quais apresentavam 169 profissionais que desempenham suas atividades nos cargos de Diretores e Educadores.

Para o cálculo do tamanho amostral, considerou-se que quando a população de referência for igual ou inferior a 250 indivíduos, recomenda-se a realização de um censo, ou seja, examina-se toda a população ${ }^{10}$. Assim, se propôs a obtenção de uma amostra de até 169 participantes.

Foram incluídos neste estudo os profissionais que durante o período da coleta dos dados concordaram em participar da pesquisa, assinando o Termo de Consentimento Livre e Esclarecido. Foram excluídos do estudo profissionais que não estiveram em atividade no período da investigação.

A coleta de dados foi realizada por dois pesquisadores. A fidedignidade das respostas foi testada pelo método de validação de "face" com $10 \%$ dos participantes. Nesse método, o pesquisador solicita aos tomadores de decisão que expliquem, com suas próprias palavras, o que entenderam sobre cada pergunta ${ }^{10}$.

Após coleta, os dados foram analisados pela estatística descritiva, utilizando o software SPSS na versão 16.0 (Statistical Package for the Social Sciences, Chicago, EUA, 2004).

Este projeto foi submetido à apreciação do Comitê de Ética em Pesquisa da Fundação Francisco Mascarenhas - Faculdades Integradas de Patos, sendo aprovado em 29 de novembro de 2012, seguindo os princípios éticos propostos na Resolução 196/96 do Conselho Nacional de Saúde e na Declaração de Helsinque.-

\section{Resultados}

Ao término da coleta de dados, 121 profissionais responderam ao questionário, o 
que representou $71,59 \%$ da amostra. Estes se caracterizaram por apresentar, em média, 40,16 anos de idade $( \pm 8,7)$ e 14,4 anos de profissão $( \pm 7,8)$. Sendo do gênero feminino $97,5 \%$.
O acessoà informaçãosobre traumatismo não foi relatado por $89,3 \%$ dos investigados (Tabela 1), não havendo diferença significativa entre os níveis de escolaridade $(p>0,05)$. Entre aqueles que obtiveram algum tipo de informação, 30,8\% citaram como meio de acesso, a Capacitação e a Leitura (Tabela 2).

Tabela 1 - Associação entre a Escolaridade dos investigados e as variáveis: acesso a informações sobre traumatismo, experiência com traumatismo, interesse sobre o tema e preparo para atuar em casos de traumatismo. Brasil, 2015.

\begin{tabular}{|c|c|c|c|c|c|c|c|}
\hline \multirow{3}{*}{ VARIÁVEIS } & \multicolumn{7}{|c|}{ ESCOLARIDADE } \\
\hline & \multicolumn{2}{|c|}{$\begin{array}{c}\text { Ensino } \\
\begin{array}{c}\text { Fundamental ou } \\
\text { Médio }\end{array}\end{array}$} & \multicolumn{2}{|c|}{$\begin{array}{c}\text { Ensino } \\
\text { Superior ou } \\
\text { Pós- } \\
\text { Graduação }\end{array}$} & \multicolumn{2}{|c|}{ Grupo Total } & \multirow[t]{2}{*}{$\begin{array}{l}\text { Valor } \\
\text { de } p\end{array}$} \\
\hline & $\mathbf{n}$ & $\%$ & $n$ & $\%$ & n & $\%$ & \\
\hline \multicolumn{8}{|c|}{$\begin{array}{l}\text { Recebeu informação/treinamento sobre } \\
\text { traumatismo? }\end{array}$} \\
\hline Sim & 5 & 4,1 & 8 & 6,6 & 13 & 10,7 & $0,760^{*}$ \\
\hline Não & 37 & 30,6 & 71 & 58,7 & 108 & 89,3 & \\
\hline Total & 42 & 34,7 & 79 & 65,3 & 121 & 100,0 & \\
\hline \multicolumn{8}{|c|}{ Preparado(a) para prestar socorro? } \\
\hline Sim & 4 & 3,3 & 8 & 6.6 & 12 & 9,9 & $0,930^{*}$ \\
\hline Não & 19 & 15,7 & 38 & 31,4 & 57 & 47,1 & \\
\hline Depende & 19 & 15,7 & 33 & 27,3 & 52 & 43,0 & \\
\hline Total & 42 & 34,7 & 79 & 65,3 & 121 & 100,0 & \\
\hline \multicolumn{8}{|c|}{$\begin{array}{l}\text { Interesse em receber informação } \\
\text { treinamento sobre traumatismo? }\end{array}$} \\
\hline Sim & 40 & 33,1 & 78 & 64,5 & 118 & 97,5 & $0,270^{\star}$ \\
\hline Não & 2 & 1,7 & 1 & 8 & 3 & 2,5 & \\
\hline Total & 42 & 34,7 & 79 & 65,3 & 121 & 100,0 & \\
\hline \multicolumn{8}{|c|}{ Alguma experiência com traumatismo? } \\
\hline Sim & 29 & 24,0 & 40 & 33,1 & 69 & 57,0 & $0,057^{*}$ \\
\hline Não & 13 & 10,7 & 39 & 32,2 & 52 & 43.0 & \\
\hline Total & 42 & 34,7 & 79 & 65,3 & 121 & 100,0 & \\
\hline
\end{tabular}

*Teste Exato de Fischer.

**Teste Qui-Quadrado de Pearson.

Tabela 2 - Distribuição dos participantes do estudo segundo o acesso as informações.

\begin{tabular}{|c|c|c|}
\hline Meio pelo qual teve acesso à informação sobre ao assunto & $\mathbf{n}$ & $\%$ \\
\hline Capacitação e Leitura. & 4 & 30,8 \\
\hline Leitura. & 3 & 23,1 \\
\hline Capacitação. & 2 & 15,4 \\
\hline Dentista, Leitura e Capacitação. & 2 & 15,4 \\
\hline Dentista. & 1 & 7,7 \\
\hline Mídia. & 1 & 7,7 \\
\hline Total & 13 & 100,0 \\
\hline
\end{tabular}


A maioria dos profissionais investigados destacou não se sentir preparados para prestar socorroàs crianças em situações de traumatismo orofacial $(47,1 \%)$, afirmando terem interesse em receber algum tipo de informação sobre o assunto (97,5\%). Estas variáveis também não estiveram associadas à escolaridade dos investigados ( $p>0,05)$. (Tabela 1).

Na Tabela 1 ainda é possível observar que $57,0 \%$ dos pesquisados vivenciou alguma situação de traumatismo na Instituição na qual trabalha, sem demonstrar associação ao nível de $5 \%$. Na tabela 3 , os traumatismos apresentaram ligação principalmente com as quedas $(55,1 \%)$, não apresentando diferenças por gênero em relação a sua ocorrência $(76,8 \%)$ e se caracterizando principalmente por serem do tipo corte ou laceração no lábio $(46,3 \%)$.

Tabela 3 - Distribuição dos participantes do estudo segundo sua experiência com traumatismo na Instituição na qual presta serviço.

\begin{tabular}{|c|c|c|}
\hline Causas de traumatismos associados aos casos vivenciados por você na Instituição & $\mathbf{n}$ & $\%$ \\
\hline Quedas & 38 & 55,1 \\
\hline Colisão Acidental & 12 & 17,5 \\
\hline Colisão Acidental e Quedas & 7 & 10,2 \\
\hline Brincadeiras no Parque & 5 & 7,2 \\
\hline Brincadeiras no Parque e Quedas & 4 & 5,8 \\
\hline Esportes & 1 & 1,4 \\
\hline Colisão Acidental e Brincadeiras no Parque & 1 & 1,4 \\
\hline Colisão Acidental, Brincadeiras no Parque e Quedas & 1 & 1,4 \\
\hline Total & 69 & 100,0 \\
\hline $\begin{array}{l}\text { Os acidentes envolvendo traumatismo que você vivenciou ocorreram mais } \\
\text { frequentemente em: }\end{array}$ & $n$ & $\%$ \\
\hline Não há diferença & 53 & 76,8 \\
\hline Menino & 16 & 13,2 \\
\hline Menina & 0 & 0,0 \\
\hline Total & 69 & 100,0 \\
\hline ipos de traumatismos associados aos casos vivenciados por você na Instituição & $\mathbf{n}$ & $\%$ \\
\hline Corte ou laceração no lábio & 32 & 46,3 \\
\hline A criança perdeu o dente & 6 & 8,7 \\
\hline Corte ou laceração na genqiva & 4 & 5,8 \\
\hline Corte ou laceração no lábio, na línqua e o dente ficou com mobilidade & 4 & 5,8 \\
\hline Corte ou laceração na genqiva e no lábio & 4 & 5,8 \\
\hline Corte ou laceração no lábio, na língua e na face & 4 & 5,8 \\
\hline Corte ou laceração na face & 3 & 4,3 \\
\hline Corte ou laceração no lábio e o dente ficou com mobilidade & 3 & 4,3 \\
\hline Corte ou laceração na línqua & 2 & 2,9 \\
\hline Dente ficou com mobilidade & 2 & 2,9 \\
\hline Corte ou laceração no lábio e línqua & 2 & 2,9 \\
\hline Dente ficou quebrado & 1 & 1,5 \\
\hline Corte ou laceração no lábio e face & 1 & 1,5 \\
\hline Todas as opções & 1 & 1,5 \\
\hline Total & 69 & 100,0 \\
\hline
\end{tabular}

Sobre a assistência prestada as crianças que frequentam as creches diante dos casos de traumatismo, foi relatado que o Professor é o primeiro profissional a prestar socorro
(59,5\%), destacando como serviço para encaminhamento dos casos de urgências com traumatismo, a Estratégia Saúde da Família $(60,0 \%)$ (Tabela 4). 
Tabela 4 - Distribuição dos participantes segundo os relatos sobre a assistência diante de situações que envolvem traumatismo na região orofacial de crianças em idade pré-escolar. Brasil, 2015.

\begin{tabular}{|c|c|c|}
\hline $\begin{array}{c}\mathrm{Na} \text { Instituição em que você trabalha em casos de urgências com traumatismo na região } \\
\text { orofacial, quem é o primeiro a prestar assistência à criança? }\end{array}$ & $\mathbf{n}$ & $\%$ \\
\hline Professor (a) & 72 & 59,5 \\
\hline Quem primeiro entrar em contato com a criança & 26 & 21,5 \\
\hline Diretor (a) & 12 & 9,9 \\
\hline Profissional de saúde ou pessoa capacitada em primeiros socorros & 8 & 6,6 \\
\hline Outro & 3 & 2,5 \\
\hline Total & 121 & 100,0 \\
\hline $\begin{array}{l}\text { Na Instituição em que você trabalha vocês contam com algum Serviço para onde } \\
\text { possam encaminhar os casos de urgências com traumatismo na região orofacial? }\end{array}$ & $\mathbf{n}$ & $\%$ \\
\hline Sim & 80 & 66,1 \\
\hline Não & 41 & 33,9 \\
\hline Total & 121 & 100,0 \\
\hline $\begin{array}{c}\text { Serviço para encaminhamento dos casos de urgências com traumatismo na região } \\
\text { orofacial }\end{array}$ & $\mathbf{n}$ & $\%$ \\
\hline Estratégia Saúde da Famíla & 48 & 60,0 \\
\hline Hospital Infantil & 20 & 25,0 \\
\hline SAMU & 12 & 15,0 \\
\hline Total & 80 & 100,0 \\
\hline
\end{tabular}

\section{A Tabela 5 apresenta o conhecimento} dos profissionais investigados sobre a relação entre dentição decídua e traumatismo em crianças em idade pré-escolar. Desta forma, $76,9 \%$ dos profissionais acreditam que os dentes decíduos são os dentes envolvidos em casos de traumatismo na região orofacial de crianças entre 3 e 5 anos de idade; considerando possível um trauma na dentição decídua comprometer a dentição permanente $(85,1 \%)$; atribuindo a mesma importância entre dentes decíduos e permanentes $(90,1 \%)$.

Tabela 5 - Distribuição dos participantes do estudo segundo o conhecimento sobre a relação entre dentição decídua e traumatismo em crianças em idade pré-escolar.

\begin{tabular}{|c|c|c|}
\hline $\begin{array}{c}\text { Em sua opinião, quais os dentes envolvidos em casos de traumatismo na região } \\
\text { orofacial de crianças entre } 3 \text { e } 5 \text { anos de idade? }\end{array}$ & $\mathbf{n}$ & $\%$ \\
\hline Decíduo & 93 & 76,9 \\
\hline Permanente & 1 & 0,8 \\
\hline Decíduo e Permanente & 8 & 6,6 \\
\hline Não sei & 19 & 15,7 \\
\hline Total & 121 & 100,0 \\
\hline $\begin{array}{c}\text { Você acha possível um trauma na dentição decídua comprometer a dentição } \\
\text { permanente? }\end{array}$ & $\mathbf{n}$ & $\%$ \\
\hline Sim & 103 & 85,1 \\
\hline Não & 15 & 12,4 \\
\hline Não sei & 3 & 2,5 \\
\hline Total & 121 & 100,0 \\
\hline Para você, o dente decíduo é tão importante quanto o dente permanente? & $\mathbf{n}$ & $\%$ \\
\hline Sim & 109 & 90,1 \\
\hline Não & 12 & 9.9 \\
\hline Total & 121 & 100,0 \\
\hline
\end{tabular}


Tabela 6 - Distribuição dos participantes do estudo segundo os cuidados exigidos diante de situações de traumatismo orofacial em crianças com idade pré-escolar.

\begin{tabular}{|c|c|c|}
\hline $\begin{array}{c}\text { Quais os primeiros cuidados que devemos ter com a criança em casos de traumatismo } \\
\text { orofacial? }\end{array}$ & $\mathbf{n}$ & $\%$ \\
\hline Limpar com qaze umedecida em soro fisiolóqico e aplicar qelo no local afetado & 49 & 40,7 \\
\hline Não sei & 32 & 26,6 \\
\hline Aplicar gelo no local afetado & 15 & 12,7 \\
\hline Limpar com gaze umedecida em soro fisiológico & 12 & 9,9 \\
\hline Não se deve fazer nada & 6 & 4,9 \\
\hline Medicar com analqésico & 3 & 2,6 \\
\hline $\begin{array}{l}\text { Limpar com gaze umedecida em soro fisiológico, aplicar gelo no local afetado e medicar com } \\
\text { analgésico }\end{array}$ & 3 & 2,6 \\
\hline Total & 121 & 100,0 \\
\hline
\end{tabular}

Quanto aos primeiros cuidados que se deve ter coma criança em casos de traumatismo orofacial, 40,7\%; dos participantes destacou que se deve limpar com gaze umedecida em soro fisiológico e aplicar gelo no local afetado (Tabela 6).

\section{Dıscussão}

Mesmo diante de uma frequência considerável de casos de traumatismos orofaciais na primeira infância e da gravidade de sua repercussão para a dentição permanente, observa-se uma carência de estudos sobre o assunto na literatura. E, apesar de a criança passar grande parte do tempo em ambiente escolar, percebe-se a ausência de investigações ligadas ao conhecimento de profissionais cuidadores de crianças em idade pré-escolar.

A observação do perfil dos participantes deste estudo demonstrou que estes são predominantemente do gênero feminino e com idade média de 40,16 anos $( \pm 8,7)$. Da mesma forma que os participantes do estudo realizado na cidade de Hong Kong, China ${ }^{11}$. $\mathrm{O}$ predomínio de profissionais do gênero feminino nas creches incluídas nesse estudo pode ser justificado pelo modelo da Educação Infantil que muito utiliza a categoria de "relações de gênero" como norteadora para o trabalho no Magistério, considerando que o cuidado e a educação das crianças pequenas têm sido uma prerrogativa das mulheres. Isto porque se convive com concepções mais ligadas ao biológico e ao forte apelo à maternidade, ou, mesmo, em alguns casos, porque o marco teórico advém de uma perspectiva voltada à política do cuidado como trabalho feminino ${ }^{12}$.

Em estudo realizado, em 2012, na cidade de Manaus, Amazonas ${ }^{13}$, o tempo de experiência profissional e o grau de formação dos Educadores foram variáveis, influenciando na conduta destes frente a situações de traumatismo orofacial, e quanto maior o tempo de atuação e o nível de formação, mais adequadas são as condutas emergenciais. Neste estudo, o tempo médio de experiência profissional dos participantes foi de $14,4( \pm 7,8)$ anos. Tempo superior àquele apresentado pelos profissionais investigados em outras situações e lugares, como em Manaus, Amazonas, em $2009^{14}$ e em Campina Grande, Paraíba ${ }^{15}$, porém inferior ao do estudo realizado em Hong Kong ${ }^{11}$. Com relação à escolaridade, constatou-se que a maior parte dos entrevistados possuía Ensino Superior completo. Resultados superiores foram encontrados na formação dos professores em estudo realizado também na cidade de Manaus $^{14}$, onde $46,7 \%$ da amostra apresentou o Ensino Superior completo.

Nesse trabalho, o acesso a informações sobre o tema foi ressaltado pela menor parte 
dos profissionais, sendo os meios de acesso; a capacitação pedagógica, a mídia, a leitura e o Cirurgião-Dentista. Esseachadofoicorroborado pelo estudo feito em Manaus ${ }^{14}$, no qual apenas $3,3 \%$ relataram terem recebido orientações quanto às situações de traumatismo orofacial; pelo estudo feito em Campina Grande ${ }^{15}$ onde apenas $13,8 \%$ das profissionais responderam que receberam orientação por meio de capacitação pedagógica, e pelo estudo feito em Manaus ${ }^{13}$, no qual apenas $12,9 \%$ dos participantes obtiveram informações sobre traumatismo dentário e facial. De forma geral, se observa um conhecimento insuficiente e atitudes falhas dos Educadores no que concerne à assistência emergencial em casos de traumas na região orofacial ${ }^{16}$.

A falta de preparo quanto ao manejo da vítima de traumatismo na região de face, em particular em relação ao trauma envolvendo dentes e estruturas de suporte são um dos aspectos responsável pelo insucesso do prognóstico de manutenção do elemento dentário ${ }^{17}$. Porém, a maioria dos profissionais investigados neste trabalho declarou não se sentir preparado para prestar os primeiros socorros em casos de traumatismo orofacial, ressaltando o interesse em participar de uma Capacitação sobre este tema, assim como apresentado por estudos realizados na Europa ${ }^{18,19}$ e na Ásia ${ }^{20}$. Porém, em discordância com os achados da pesquisa realizada em Campina Grande ${ }^{15}$, na qual a maioria dos profissionais se considera apta a intervir diante de um caso de traumatismo.

Segundo relato dos profissionais questionados, os traumatismos por eles vivenciados estiveram associados principalmente às quedas. Os mesmos não perceberam diferenças por gênero em relação a sua ocorrência. Segundo estudo realizado na cidade de Recife, Pernambuco ${ }^{21}$ crianças entre 1 e 4 anos de idade estão consolidando a marcha e ainda não têm reflexos completamente desenvolvidos, sendo então, mais susceptíveis às quedas e as colisões acidentais; apontadas como os fatores causais predominantes do traumatismo orofacial ${ }^{3,22}$. Em relação ao gênero, a literatura é bastante controversa; citando-se que o gênero masculino prepondera ${ }^{3,23,24}$; ou que em crianças não há diferença na prevalência de traumatismos entre os gêneros ${ }^{25}$. Entre as lesões observadas pelos profissionais participantes estes citaram principalmente corte ou laceração no lábio; corroborando aos resultados encontrados no estudo feito em Campina Grande ${ }^{15}$.

Os indivíduos citados pelos participantes como primeiros prestadores de assistência à criança em casos de traumatismo foram os Professores, dado este confirmado pela pesquisa feita em Campina Grande ${ }^{15}$. Nesta perspectiva, apesar de rotineiramente situações que envolvem esse tipo de acidente serem irrelevantes e culturalmente pouco importantes, convém lembrar que é o Professor o profissional que está com a tutela da criança durante o expediente escolar, devendo estar bem preparado para agir de maneira eficaz nestas situações ${ }^{14}$. Desta forma, os mesmos autores ${ }^{13}$ destacam que estratégias para desenvolver competências acerca dos traumatismos orofaciais deveriam ser inseridas na formação desses profissionais.

Nas creches avaliadas, em casos de emergência, as crianças são encaminhadas a Unidade Básica de Saúde da Estratégia de Saúde da Família mais próxima, atitude diferente daquela citada pelos profissionais do estudo realizado em Manaus ${ }^{14}$, no qual os profissionais relataram que encaminhariam as crianças acidentadas para a enfermaria da escola; e em Campina Grande ${ }^{15}$, cujos profissionais encaminhariam para o hospital.

Questionadas sobre quais os dentes envolvidos em casos de traumatismo orofacial em crianças de 1 a 5 anos de idade, a maior parte dos participantes destacou ser o dente 
decíduo. Os participantes ressaltaram ainda que um trauma no dente decíduo pode comprometer a dentição permanente e que o dente decíduo é tão importante quanto o dente permanente. Há uma relação muito estreita entre o dente decíduo afetado por um trauma e seu sucessor dente permanente ${ }^{22}$.

Por fim, questionou-se aos participantes sobre quais os primeiros cuidados que se deve ter com a criança em casos de traumatismos orofaciais. Estes destacaram principalmente a limpeza com gaze umedecida em soro fisiológico e a aplicação de gelo no local afetado; dados diferentes daqueles obtidos no estudo realizado na cidade de Campina Grande ${ }^{15}$, onde a maior parte dos entrevistados afirmou que realizaria "outros procedimentos", sem especificações quanto à conduta. Segundo estudo feito na cidade de Curitiba, Paraná22, a limpeza da região injuriada é uma das primeiras medidas recomendadas para situações de traumatismo orofacial, aspecto que contribui para a redução do risco de infecção no local afetado.

Considerando que o binômio educarcuidar representa um dos principais aspectos do trabalho na educação infantil, os dados observados neste estudo implicam reflexões sobre a necessidade de capacitação dos profissionais de creches, para agirem de maneira adequada diante de situações de traumatismos orofaciais. Desta forma, estes poderão apresentar uma participação mais efetiva no que concerne aos primeiros cuidados que se deve adotar diante de acidentes com crianças na primeira infância, contribuindo para um melhor prognóstico após intervenção do profissional da Odontologia.

Nesta perspectiva, destaca-se a demanda por maiores esclarecimentos e treinamento dos profissionais da educação que trabalham com crianças de pouca idade sobre a conduta diante de lesões traumáticas na dentição decídua.-

\section{Conclusão}

A Equipe de Educação Infantil investigada apresentou um conhecimento razoável e com algumas limitações com relação ao traumatismo orofacial em crianças com idade pré-escolar, o que desperta para a necessidade de uma abordagem educativa junto a estes profissionais sobre o assunto.

\section{REFERÊNCIAS}

1.CURYLOFO, P. A.; LORENCETTI, K. T.; SILVA, S. R. C. Avaliação do conhecimento de professores sobre avulsão dentária. Arq Odontol.; v.48,n.3,p.175-80.2012.

2 GLENDOR, U. Epidemiology of traumatic dental injuries-a 12 year review of the literature. Dent Traumatol; v.24,n.6,p.603-611, 2008.

3.COSTA, L. E. D.; QUEIROZ, F. S.; NÓBREGA, C. B. C.; et al.,. Trauma dentário na infância: avaliação da conduta dos educadores de creches públicas de Patos-PB. Rev Odonto UNESP; v.43,n.6,p.402-408,2014.;

4.ARAGÃO, A. K. R.; SOUSA, P. G. B.; FERREIRA, J. M. S.; et al.,. Conhecimento de professores das creches municipais de João Pessoa sobre saúde bucal infantil. Pesq Bras Odontoped Clín Integr; v.10,n.3,p.393-398,2010.

5.ANDREASEN, J. O.; LAURIDSEN, E.; GERDS, T. A.; et al,. Dental trauma guide: a source of evidence-based treatment guidelines for dental trauma. Dent Traumatol; v.28,n.2,p.345-350,2012.

6 HASHIM, R. Dental trauma management awareness among primary school teachers in the Emirate of Ajman, United Arab Emirates. Eur J Paediatr Dent; v.12,n.2,p.99-102,2011.

7 SIQUEIRA, A. C.; GONÇALVES, P. E. Avulsão dentária traumática acidental: cuidados odontológicos para o reimplante. Rev. Fac. Odontol. Lins; v.22,n.1,p.47-53,2012.

8.BERTI, M.; FURLANETTO, D. L. C.; REFOSCO, M.Z. Avaliação do conhecimento de professores do ensino fundamental sobre o tema avulsão dentária. Pesq Bras Odontoped Clín Integr; v.11,n3,p.381-386,2011. 
9 ALVES, L. S. B.; FREITAS, V. J. G.; ROSENDO, R. A.; et al,. Avaliação do conhecimento de professores do ensino fundamental da rede particular sobre atendimento imediato de vítima de traumatismo dental. RFO; v.20,n.3,p.302-307, 2015.

10 ANTUNES, J. L. F.; PERES, M. A. O método epidemiológico de investigação e sua contribuição para a saúde bucal. In: Fundamentos de Odontologia: Epidemiologia da Saúde Bucal. Rio de Janeiro: Guanabara Koogan, 2006.

11 YOUNG, C.; WONG, K. Y.; CHEUNG, L.K. Emergency management of dental trauma: knowledge of Hong Kong primary and secondary school teachers. Hong Kong Med J; v.18,n.5,p.362-370, 2012.

12 SAYÃO DT. Relações de gênero e trabalho docente na educação infantil: Um estudo de professores em creche. Tese (Doutorado em Educação) - Centro de ciências da Educação. Universidade Federal de Santa Catarina Centro de ciências da educação programa de PósGraduação em Educação, Santa Catarina, 2005.

13 HANAN, A. S.; COSTA, S. K. Conhecimento dos Professores de $1^{\text {a }}$ a $4^{\text {a }}$ Série de Escolas Públicas Municipais de Manaus/AM Frente à Avulsão Dentária. Pesq Bras Odontop Clín Integr; v.10,n.1,p.27-33, 2010.

14 SILVA, M. B.; COSTA, A. M. M.; ALMEIDA, M. E. C.; et al., Avaliação do conhecimento da abordagem de trauma dental, pelos profissionais de creches. Conscientia e Saúde;v.8,n.1,p.65-73, 2009.

15 CORDEIRO, P. M.; FONTES, L. B. C.; GRANVILKE-GARCIA, A. F.; et al., Percepção de diretores, professores e berçaristas de creches públicas sobre traumatismos orofaciais. Rev Odontol UNESP;v.39,n.3,p.169-173, 2010. 16 SILVA, E. D.; SIQUEIRA, M. F. G.; GOMES, M. C.; et al., Conhecimento de alunos do curso de Educação Física sobre avulsão dentária. Arq Odontol; v.49,n.4,p.177-183, 2013.; 49(4): 177-83.
17 BITTENCOURT, A. M.; PESSOA, O. F.; DA SILVA, J. M. Avaliação do conhecimento de professores em relação ao manejo da avulsão dentária em crianças. Rev Odontol UNESP; v.37,n.1,p.15-19, 2008.

18 BLAKYTNY, C.; SURBUTS, C.; THOMAS, A.; et al., Avulsed permanent incisors: knowledge and attitudes of primary school teachers with regard to emergency management. Int J Paed Dent; v.11,n.5,p.327-332, 2001.

19 ÇAGLAR, E.; FERREIRA, L. P.; KARGUE, B. Dental trauma management knowledge among a group of teachers in two South European Cities. Dent Traumatol; v.21,n.5,p.258-262,2005.

20 SAE-LIM, V.; LIM, L. P. Dental Trauma management awareness of Singapore pre-school teachers. Dent Traumatol; v.17,n.2,p.71-76, 2001.

21 GARCIA, A. F. G.; MENEZES, V. A.; LIRA, P. I. C. Prevalência e fatores sociodemográficos associados ao traumatismo dentário em pré-escolares. Odontologia. Clín.Científ;v.5,n.1p.57-64, 2006.

22 LOSSO, E. M.; TAVARES, M. C. R.; BERTOLI, F. M. P.;et al.Dentoalveolar trauma in the primary dentition. RSBO;v.8,n.1,p.1-18, 2011.

23 CARDOSO, M.; CARVALHO ROCHA, M. J. Traumatized primary teeth in children assisted at the Federal University of Santa Catarina, Brazil. Dent Traumatol;v.18,n.3,p.129-133, 2002.

24 KRAMER, P. F.; ZEMBRUSKI, C.; FERREIRA, S. H.;et al. Traumatic dental injuries in Brazilian preschool children. Dent Traumatol;v.19,n.6,p.299-303, 2003.

25 MESTRINHO, H. D.; BEZERRA, A. C.; CARVALHO, J. C. Traumatic dental injuries in Brazilian pre-school children. Braz Dent J;v.9,n.2,p.101-104, 1998.

Submetido em: 101-6-2016

Aceito em: 22-8-2016 